\title{
AJUSTES AOS US-GAAP: ESTUDO EMPÍRICO SOBRE SUA RELEVÂNCIA PARA EMPRESAS BRASILEIRAS COM ADRs NEGOCIADOS NA BOLSA DE NOVA IORQUE*
}

\section{ADJUSTMENTS TO US-GAAP: EMPIRICAL STUDY ON THEIR RELEVANCE FOR BRAZILIAN COMPANIES WITH ADR TRADED ON THE NEW YORK STOCK EXCHANGE}

\author{
FÁBIO MORAES DA COSTA \\ Professor Associado da Fundação Instituto Capixaba \\ de Pesquisas em Contabilidade, Economia e Finanças - ES \\ E-mail:fabio@fucape.br
}

\author{
ALEXSANDRO BROEDEL LOPES \\ Professor Livre-Docente do Departamento de Contabilidade e Atuária \\ da Faculdade de Economia, Administração e Contabilidade \\ da Universidade de São Paulo - SP \\ Pesquisador do CNPq \\ E-mail: broedel@usp.br
}

\section{RESUMO}

A relevância das informações disponibilizadas ao mercado por empresas brasileiras com American Depositary Recepts (ADRs) negociados na Bolsa de Nova Iorque foi avaliada neste trabalho. Essas empresas devem atender à exigência da Securities and Exchange Commission e elaborar suas demonstrações contábeis de acordo com os United States Generally Accepted Accounting Principles (US-GAAP) até 30 de junho, anualmente. Diante desse fato, dois conjuntos de informações estão disponíveis, promovendo a base de dados para o estudo da relevância das variáveis lucro líquido e patrimônio líquido para o período entre 1999 e 2003. Após a utilização do modelo desenvolvido por Ohlson (1995) e de seus desdobramentos posteriores, os resultados evidenciam que (1) as informações elaboradas segundo os princípios de contabilidade geralmente aceitos no Brasil são relevantes; (2) as informações em US-GAAP disponibilizadas em junho possuem menor ou igual relevância do que as divulgadas em abril e (3) ọ ajustes aos US-GAAP efetuados no patrimônio líquido são relevantes para o mercado de capitais brasileiro.

Palavras-chave: Contabilidade Internacional; Mercado de Capitais; ADR; Modelo de Ohlson.

\section{ABSTRACT}

This study evaluated the value-relevance of the information disclosed by Brazilian companies with American Depositary Receipts traded on the New York Stock Exchange. Those companies must comply with the requirement of the Securities and Exchange Commission and elaborate their financial statements according to the United States Generally Accepted Accounting Principles (US-GAAP) until $30^{\text {th }}$ of June, annually. Thus, two sets of information are available, providing data to evaluate the value-relevance of the variables net income and stockholders' equity, from 1999 to 2003. After applying the model Ohlson developed in 1995 and its further developments, results indicate that (1) the information elaborated according to the generally accepted accounting principles in Brazil are value-relevant; (2) the relevance of the information in US-GAAP made available in June is lower or equal to that disclosed in April; and (3) the adjustments to US-GAAP for stockholders' equity are value-relevant to the Brazilian capital market.

Keywords: International Accounting; Capital Markets; ADR; Ohlson's Model. 


\section{INTRODUÇÃO}

Ao longo dos séculos, a Contabilidade vem sendo utilizada como instrumento de apoio à tomada de decisão, por consistir em um modelo de identificação, mensuração e divulgação dos eventos econômicos que afetam uma entidade.

Ball, Kothari e Robin (2000) constatam que os profissionais de mercado e acadêmicos começaram a se interessar pela análise das diferenças entre os modelos contábeis, devido ao movimento contínuo de internacionalização das atividades empresariais.

Atualmente, o organismo comumente apontado como o responsável pela coordenação e realização de esforços em prol da convergência dos pronunciamentos contábeis é o International Accounting Standards Board (IASB) (BARTH et al.,1999).

Recentemente, foi firmado um memorando de entendimento entre o organismo norte-americano FASB (Financial Accounting Standards Board) e o IASB, conhecido como "The Norwalk Agreement". Espera-se que o acordo entre os organismos resulte em dois modelos contábeis similares dentro de alguns anos.

Ainda há, relativamente, poucas evidências empíricas sobre a aproximação entre as normas norte-americanas e internacionais e seus efeitos no mercado de capitais. Destaque-se, porém, o estudo de Haverty (2004), o qual apresenta resultados que confirmam o maior grau de aproximação entre as normas nos últimos anos. Além disso, segundo Leuz (2003), não haveria subsídios para afirmar que um conjunto de princípios contábeis teria maior conteúdo informacional do que o outro.

Considerando a informação contábil como uma sinalização válida para os usuários externos, seu processo de regulamentação teria como uma de suas justificativas a incapacidade dos indivíduos em distinguirem empresas eficientes das ineficientes (LEFTWICH, 1980).

Dado que a regulação incorre em custos adicionais a seus usuários, o processo de elaboração ou mudança de um pronunciamento contábil está exposto a pressões de cada grupo afetado. Nesse sentido, a elaboração de um modelo contábil teria seu desenvolvimento influenciado pelo complexo relacionamento entre os agentes do mercado, os quais, direcionados pelas conseqüências econômicas de cada pronunciamento ou normatização, realizariam lobby em interesse próprio (ZEFF, 1994).

Como resultado, o que justificaria a existência de diferenças entre os modelos contábeis de cada país estaria relacionado à estrutura de relacionamento entre grupos de usuários e os seus respectivos poderes de influência no processo de elaboração, discussão e modificação de pronunciamentos e normatizações. Daí a discrepância entre os conjuntos de Princípios Contábeis Geralmente Aceitos (PCGAs) entre os países.

Como um dos fatores fundamentais em termos de estimativas de risco e de retorno corresponde à qualidade dos inputs, entendida como a utilização de parâmetros adequa- dos de estimação, o conteúdo informacional constante nas demonstrações contábeis divulgadas corresponde a um dos parâmetros relevantes levados em consideração.

Nesse sentido, estudos foram desenvolvidos para avaliar os impactos em países cujas empresas passaram a divulgar dois grupos de demonstrações contábeis, notadamente segundo o modelo norte-americano, de acordo com os US-GAAP (United States Generally Accepted Accounting Principles) e mais recentemente, em conformidade com os IFRS (International Financial Reporting Standards) emitidos pelo IASB. As pesquisas empíricas apresentam resultados conflitantes sobre a maior ou menor relevância da informação contábil quando um novo GAAP é adotado.

Analiticamente, o modelo desenvolvido por Barth et al. (1999) propõe-se a demonstrar porque a convergência entre normas contábeis não traria, necessariamente, apenas benefícios, devendo-se levar em consideração dois efeitos: impacto no erro de mensuração do valor da companhia contido nas demonstrações contábeis e suas conseqüências nos custos e benefícios para um investidor estrangeiro adquirir ou não conhecimento acerca do GAAP doméstico.

Em linhas gerais, dependendo de como a convergência altere o erro de mensuração do valor da companhia (aumento ou diminuição), esse influenciaria diretamente os custos e benefícios de obtenção de conhecimento do GAAP doméstico pelos investidores estrangeiros (aumento ou diminuição). Dessa forma, nem sempre seria benéfica a redução das diferenças de tratamento contábil entre os países, devendo-se analisar cada país individualmente (BARTH et al., 1999).

Dentro desse contexto, enquadra-se a discussão proposta no presente trabalho: avaliar se a informação contábil baseada em uma outra normatização, que não a do país nativo, seria relevante ou não ao mercado de capitais.

Para tanto, foram identificadas empresas brasileiras que possuem e divulgam regularmente dois conjuntos de demonstrações contábeis: um baseado nos PCGAs brasileiros e outro de acordo com os US-GAAP. Tais empresas compreendem aquelas que possuem American Depositary Receipts (ADRs) negociados na Bolsa de Nova lorque, pois a SEC (Securities and Exchange Commission) exige que tais empresas forneçam suas informações contábeis baseadas nos pronunciamentos contábeis norte-americanos.

A exigência do órgão normatizador do mercado de capitais norte-americano enseja questionamentos em relação ao custo das empresas estrangeiras em realizar a reelaboração de suas demonstrações contábeis e o benefício informacional que seria obtido de tais informações aos seus usuários. Tal fato, portanto, incorre na necessidade de constatação empírica se realmente há nova informação relevante para o mercado ou se as demonstrações contábeis realizadas conforme a norma local seriam suficientes para a avaliação dos investidores.

Além disso, possibilita-se a avaliação da relevância da informação elaborada segundo um conjunto de princípios 
contábeis desenvolvidos em um país cujas características são contrastantes com as observadas no modelo contábil brasileiro.

Assim, o estudo buscará subsídios que proporcionem a adequada fundamentação para responder à seguinte ques- tão de pesquisa: Os ajustes aos US-GAAP, realizados anualmente por empresas brasileiras com ADRs negociados na Bolsa de Nova lorque, são relevantes para o mercado de capitais brasileiro?

\section{REVISÃO DA LITERATURA}

Até o início da década de 80, a maior parte das pesquisas que relacionavam variáveis contábeis e de mercado era focada em mercados desenvolvidos, principalmente nos Estados Unidos e no Reino Unido.

Um dos primeiros estudos relacionando empresas domésticas e estrangeiras foi realizado por Meek (1983), ao comparar a reação do mercado norte-americano a informações de empresas baseadas em normas contábeis de seus respectivos locais de origem versus a de empresas norte-americanas, elaboradas em conformidade com os US-GAAP. A conclusão do estudo é a de que o mercado de capitais norte-americano reage às divulgações de resultados de empresas, mesmo que estes não sejam baseados nos US GAAP (MEEK,1983, p. 394).

Meek e Thomas (2004) destacam que nas últimas duas décadas houve o aumento e a diversificação de pesquisas em Contabilidade Internacional que utilizavam variáveis de mercado (preço e volume, por exemplo). Os focos de tais trabalhos consistem em comparações entre países; aplicações em algum país específico, mas com influências em outros; avaliação de empresas estrangeiras e estudos envolvendo atividades de operação e captação de multinacionais.

Como o foco do presente trabalho consiste em avaliar a relevância da informação contábil divulgada segundo os US-GAAP por empresas brasileiras com ADRs negociados na Bolsa de Nova lorque, a seguir serão comentadas as principais pesquisas já desenvolvidas nessa linha.

Choi e Levich (1991) chegaram à conclusão de que o entendimento das diferenças entre princípios contábeis é relevante para a tomada de decisão, mas que a elaboração de uma nova demonstração (restatement) não seria capaz de eliminar toda a diversidade de tratamento. Bhushan e Lessard (1992) concluíram que os ajustes seriam desejáveis para os investidores, e não indispensáveis nas análises.

Diante do debate concernente às adaptações a GAAPs distintos, testes empíricos foram realizados com o intuito de avaliar se essas seriam relevantes ou não para o mercado. Porém, os resultados encontrados apresentam evidências pró e contra os ajustes, incorrendo em um campo de pesquisas ainda fértil, dada a quantidade de resultados contraditórios, não permitindo uma conclusão generalizada sobre o tema (MEEK e SAUDAGARAN, 1997; MEEK e THOMAS, 2004).

Pownall (1993) afirma que estudos são necessários para avaliar se o GAAP doméstico promoveria informa- ções em quantidade suficiente, mesmo para investidores que baseiam suas decisões nos US-GAAP, sob a hipótese de que as informações relevantes poderiam ser inferidas a partir da demonstração contábil doméstica.

Nesse sentido, Alford et al. (1993) realizaram um estudo acerca da relevância de resultados divulgados em vários países comparando-os com uma amostra de empresas norte-americanas (matched sample). O trabalho conclui que informações divulgadas por empresas australianas, francesas, holandesas e inglesas são mais relevantes que as apresentadas nos Estados Unidos. Por outro lado, os resultados de empresas da Dinamarca, Alemanha, Itália, Singapura e Suécia apresentaram menor relevância.

Amir, Harris e Venuti (1993) focaram seus esforços em analisar a relevância das reconciliações preenchidas nos formulários 20-F1 depositados na SEC, elaboradas para ajustar as demonstrações contábeis de 20 países aos US-GAAP. Os autores concluem que as reconciliações possuem relevância incremental às informações baseadas no GAAP doméstico.

Por sua vez, Bandyopadhyay, Hanna e Richardson (1994) concluem que as diferenças entre os GAAPs do Canadá e Estados Unidos não seriam relevantes para os investidores. Porém, os autores destacam que sua metodologia não permite afirmar que as reconciliações não seriam relevantes, alegando que tal fato estaria além de seu foco de estudo, sugerindo a avaliação da necessidade de reconciliações ante os seus custos de elaboração.

Harris, Lang e Möller (1994) realizaram um estudo aplicado a empresas do mercado alemão. A conclusão do estudo é a de que os lucros líquidos calculados segundo as normas alemãs teriam relevância equivalente aos calculados segundo os US-GAAP. Entretanto, em relação aos patrimônios líquidos divulgados, o poder explicativo conforme os US-GAAP é superior ao conforme os princípios alemães. Os autores destacam que os ajustes realizados aos US-GAAP possuiriam relevância incremental para o mercado em relação ao patrimônio líquido.

Chan e Seow (1996) introduziram uma nova metodologia de pesquisa, ao avaliar a relevância das informações adaptadas aos US-GAAP de empresas de 13 países diferentes. Por meio de um teste estatístico para avaliar a diferença de poder explicativo das regressões, foram comparados os valores informacionais dos lucros líquidos e dos patrimônios líquidos informados segundo os princípios do país de 
origem versus os norte-americanos. Os autores concluem que as informações domésticas são mais relevantes do que as elaboradas segundo a exigência da SEC.

Barth e Clinch (1996) realizaram um estudo em empresas australianas, britânicas e canadenses, avaliando a relevância das diferenças entre os conjuntos de princípios domésticos e sua reconciliação para os US-GAAP. A conclusão é a de que a exigência da SEC em relação às reconciliações representaria informação útil e relevante para os investidores de empresas australianas e britânicas; porém, tal benefício teria um menor impacto para empresas canadenses.

Davis-Friday e Rivera (2000) avaliaram a relevância das diferenças entre a contabilização mexicana e a norte-americana. Levando-se em conta os aspectos gerais, os resultados demonstram que o ajuste aos US-GAAP não seria relevante para os investidores, sob a justificativa de que eles considerariam o GAAP doméstico (mexicano) como suficiente para realizar suas análises.

Tomando-se a literatura de estudos que procuram avaliar a relevância como um todo, percebe-se que ainda há oportunidades a serem exploradas. Pope (1993) destaca que pesquisas que utilizam amostras de empresas de di- versos países em comparação com os US-GAAP assumem a questionável hipótese de que os GAAPs domésticos seriam homogêneos. O autor ressalta que isso poderia afetar o resultado dos testes.

Meek e Thomas (2004) ressaltam que as pesquisas deveriam ser estendidas a outros países. Os autores destacam que a maioria dos estudos já desenvolvidos baseia-se em amostras de empresas da Austrália, Canadá e/ou Reino Unido, países que seriam considerados como commonlaw. Apenas os trabalhos de Harris, Lang e Möller (1994) e de Davis-Friday e Rivera (2000) seriam aplicados especificamente a países considerados como code-law².

Assim, o presente trabalho contribui para a literatura ao incluir uma amostra composta, exclusivamente, por empresas brasileiras para o estudo sobre a relevância de ajustes aos US-GAAP. Ressalte-se que essas consistem na terceira colocação em termos de quantidade de companhias por país com ADRs negociados na Bolsa de Nova lorque, com 33 programas ao final de 2004. Na primeira colocação estão as empresas canadenses (76 programas), seguidas pelas britânicas (50 programas).

\section{METODOLOGIA DE PESOUISA}

No presente estudo, será empregada a metodologia positiva em Contabilidade, visando proporcionar a isenção ou ao menos a redução de julgamentos por parte do autor (FRIEDMAN, 1966; WILBER, 1975).

Neste trabalho, portanto, procurou-se obter subsídios que possibilitassem responder ao seguinte questionamento:

Os ajustes aos US-GAAP, realizados anualmente por empresas brasileiras com ADRs negociados na Bolsa de Nova lorque, são relevantes para o mercado?

Barth, Beaver e Landsman (2001) afirmam que o modelo amplamente utilizado nos estudos acerca da relevância de variáveis contábeis corresponde ao desenvolvido por Ohlson (1995) e suas posteriores atualizações (FELTHAM e OHLSON, 1995; FELTHAM e OHLSON, 1996).

Utilizando a dinâmica da informação linear, o modelo de Ohlson (1995) é reapresentado relacionando o valor de mercado da empresa com as variáveis patrimônio líquido e lucro líquido do período, permitindo o teste da relevância de tais informações geradas pela Contabilidade para o mercado. Assim:

$$
P_{i t}=\theta_{o}+\theta_{1} P L_{i t}+\theta_{2} L u c_{i t}+\varepsilon_{i t}
$$

em que:

$$
\begin{aligned}
\mathbf{P}_{\mathbf{i t}=} & \text { preço das ações da empresa i na data máxima } \\
& \text { permitida para publicação das demonstrações } \\
& \text { contábeis após o final do exercício social t } t^{3} ;
\end{aligned}
$$

$\mathbf{P L}_{\mathbf{i t}} \quad=$ patrimônio líquido por ação da empresa i no final do exercício social t;

Luc $_{\text {it }}=$ lucro líquido por ação da empresa i no final do período te

$\varepsilon_{\mathrm{it}} \quad=$ termo de erro da regressão.

Para que o lucro líquido e o patrimônio líquido sejam testados separadamente, adotou-se a proposição de Collins et al. (1997) para efetuar o desmembramento da equação (2) anteriormente apresentada. Dessa forma, duas equações podem ser testadas:

$$
\begin{gathered}
P_{i t}=\delta_{o}+\delta_{l} P L_{i t}+\varepsilon_{i t} \\
P_{i t}=\delta_{2}+\delta_{3} L u c_{i t}+\varepsilon_{i t}
\end{gathered}
$$

Em relação ao tratamento das variáveis utilizadas nas regressões, Brown et al. (1999) sugerem o seu deflacionamento para que não haja efeitos na escala econométrica que possam levar a distorções no dimensionamento de seu poder explicativo $\left(R^{2}\right)$. Os autores apresentam, como solução, a divisão de todas as variáveis de cada equação pelo preço da ação no período anterior $\left(\mathrm{P}_{\mathrm{it}-1}\right)$.

Para fins deste trabalho, será utilizado o valor de mercado de cada empresa, similarmente ao estudo desenvolvido por Davis-Friday e Rivera (2000). Dessa forma, dado

\footnotetext{
2 La Porta et al. (1997; 1998; 2000) e Ball et al. (2000) apresentam as principais bases de diferenciação entre países considerados common-law e code-law: sistema financeiro, oportunidade da informação e conservadorismo.

3 No Brasil, as empresas têm o prazo de 4 meses para publicarem suas demonstrações contábeis. No caso de informações em US-GAAP, permite-se um prazo para depósito do formulário 20-F na SEC até junho.
} 
que as equações anteriores foram elaboradas na base "por ação", seus termos serão substituídos pelo valor total das ações, assim como o deflacionamento das variáveis pelo valor de mercado no ano anterior, conforme sugerido por Brown et al. (1999).

A discussão metodológica, tomada em conjunto com a revisão de trabalhos anteriores, elaborada no capítulo anterior, permite a definição das equações que serão testadas empiricamente.

Para obter o embasamento necessário para responder à questão de pesquisa do trabalho, três hipóteses foram desenvolvidas:

$\mathbf{H}_{1 \mathrm{a}}=$ preços das ações em abril podem ser explicados, com coeficientes estatisticamente significativos, por variáveis contábeis elaboradas segundo os PCGAs brasileiros.

$\mathbf{H}_{1 \mathrm{~b}}=$ preços das ações em junho podem ser explicados, com coeficientes estatisticamente significativos, por variáveis contábeis elaboradas segundo os US-GAAP.

$\mathbf{H}_{1 \mathrm{c}}=$ preços em junho podem ser explicados, com coeficientes estatisticamente significativos, pela parcela do lucro líquido e do patrimônio líquido não explicada pelas demonstrações elaboradas segundo os PCGAs brasileiros.

Para que seja possível testar a relevância das informações contábeis elaboradas segundo o conjunto de princípios brasileiros, serão utilizadas as equações (5) e (6), avaliando o poder explicativo do lucro líquido e do patrimônio líquido, respectivamente. Assim, será possível responder à primeira hipótese do presente trabalho $\left(\mathbf{H}_{\mathbf{l a}}\right)$ :

$$
\begin{aligned}
& V M a b_{i t}=\alpha_{o}+\alpha_{1} \operatorname{Lucbr}_{i t}+\varepsilon_{i t} \\
& V M a b_{i t}=\alpha_{2}+\alpha_{3} P L b r_{i t}+\varepsilon_{i t}
\end{aligned}
$$

em que:
$\mathbf{V M a b}_{\mathbf{i t}}=$ valor de mercado da empresa i 4 meses após o exercício social t;
Lucbr $_{\text {it }}=$ lucro líquido da empresa i calculado segundo os PCGAs brasileiros para o exercício social $\mathrm{t}$;
$\mathbf{P L b}_{\mathbf{i t}}=$ patrimônio líquido da empresa i calculado se- gundo os PCGAs brasileiros para o exercício social t e
$\varepsilon_{\text {it }} \quad=$ termo de erro da regressão.

A segunda hipótese do trabalho a ser testada corresponde à relevância das informações contábeis elaboradas segundo os US-GAAP para o mercado $\left(\mathbf{H}_{1 b}\right)$. As equações a serem testadas são similares às apresentadas para a pri- meira hipótese, exceto pela data-base utilizada (preços de junho). Assim:

$$
\begin{gathered}
V M j u_{i t}=\lambda_{o}+\lambda_{1} \text { Lucus }_{i t}+\varepsilon_{i t} \\
V M j u_{i t}=\lambda_{2}+\lambda_{3} \text { PLus }_{i t}+\varepsilon_{i t}
\end{gathered}
$$

em que:

$\mathbf{V M j u}_{\mathbf{i t}}=$ valor de mercado da empresa i 6 meses após o exercício social t;

Lucus $_{\text {it }}=$ lucro líquido da empresa i calculado segundo os US-GAAP para o exercício social t;

PLus $_{\mathbf{i t}}=$ patrimônio líquido da empresa i calculado segundo os US-GAAP para o exercício social t e

$\varepsilon_{\mathbf{i t}} \quad=$ termo de erro da regressão.

Porém, dado que há similaridades entre o tratamento contábil segundo os PCGAs brasileiros e os US-GAAP, espera-se que as regressões (7) e (8) não possuam valor explicativo maior que as informações seguindo os PCGAs brasileiros na mesma data. Tal fato é justificado porque as variáveis contábeis em US-GAAP refletiriam informações antigas (divulgadas e/ou antecipadas em abril) e novas.

Dessa forma, com o objetivo de se comprovar a assertiva do parágrafo anterior, será utilizada a regressão para comparação entre as informações entre os princípios diferenciados, similarmente ao realizado por Chan e Seow (1996).

Portanto, as regressões (9) e (10) serão utilizadas para testar o lucro líquido e o patrimônio líquido conforme os PCGAs brasileiros, comparando ${ }^{4}$ os resultados com as regressões (7) e (8), respectivamente. Assim:

$$
\begin{aligned}
& V M j u_{i t}=\varphi_{o}+\varphi_{1} L u c b r_{i t}+\varepsilon_{i t} \\
& V M j u_{i t}=\varphi_{2}+\varphi_{3} P L b r_{i t}+\varepsilon_{i t}
\end{aligned}
$$

em que:

$\mathbf{V M j u}_{\mathbf{i t}}=$ valor de mercado da empresa i 6 meses após o exercício social t;

Lucbr $_{\text {it }}=$ lucro líquido da empresa i calculado segundo os PCGAs brasileiros para o exercício social t;

$\mathbf{P L b}_{\text {it }}$ = patrimônio líquido da empresa i calculado segundo os PCGAs brasileiros para o exercício social t $e$

$\varepsilon_{\mathbf{i t}} \quad=$ termo de erro da regressão.

Para que se possa avaliar se as informações obtidas das demonstrações contábeis elaboradas em US-GAAP refletiriam informações novas e relevantes ao mercado, os dados serão tratados em dois estágios: no primeiro, serão utiliza-

\footnotetext{
4 Como o trabalho será elaborado utilizando-se dados combinados (pooled data), fica impossibilitada a aplicação de testes de diferença de poder explicativo entre as equações, tais como o proposto por Vuong (1989). Dessa forma, os resultados corresponderão a indicativos acerca da relevância das informações elaboradas em US-GAAP em relação àquelas segundo os PCCAs brasileiros.
} 
das equações para regressão entre as variáveis lucro líquido e patrimônio líquido segundo o modelos norte-americano e brasileiro; no segundo, os resíduos de tais regressões serão utilizados como proxies para novas informações em USGAAP, pois refletem a parcela não explicada pelo modelo brasileiro, permitindo o teste empírico da terceira hipótese do trabalho $\left(\mathbf{H}_{1 \mathrm{c}}\right)$.

Portanto, as regressões (11) a (14) permitem o teste empírico da terceira hipótese do trabalho. Para testar a relevância do Lucro Líquido tem-se que:

$$
\begin{gathered}
\operatorname{Lucus}_{i t}=\psi_{0}+\psi_{1} \operatorname{Lucbr}_{i t}+\varepsilon_{i t} \\
\operatorname{PLus}_{i t}=\psi_{5}+\psi_{6} P L b r_{i t}+\varepsilon_{i t}
\end{gathered}
$$

em que:

$$
\begin{aligned}
\text { Lucus }_{\mathbf{i t}}= & \text { lucro líquido da empresa i calculado segundo } \\
& \text { os US-GAAP para o exercício social t; } \\
\text { Lucbr }_{i \mathbf{i t}}= & \text { lucro líquido da empresa i calculado segundo } \\
& \text { os PCGAs brasileiros para o exercício social t; } \\
\mathbf{P L u s}_{\mathbf{i t} \mathbf{}}= & \text { patrimônio líquido da empresa i calculado se- } \\
& \text { gundo os US-GAAP para o exercício social t; } \\
\mathbf{P L b r}_{\mathrm{it}}= & \text { patrimônio líquido da empresa i calculado se- } \\
& \text { gundo os PCGAs brasileiros para o exercício } \\
& \text { social t e } \\
= & \text { termo de erro da regressão (parte do lucro lí- } \\
& \text { quido ou do patrimônio líquido em US-GAAP } \\
& \text { não explicada pelo lucro líquido segundo os } \\
& \text { PCGAs brasileiros). }
\end{aligned}
$$

Em seguida, os termos de erro das regressões (11) e (12) serão utilizados, respectivamente, nas equações (13) e (14), para avaliar a relevância do ajuste do lucro líquido e do patrimônio líquido aos US-GAAP. Dessa forma:

$$
\begin{aligned}
& V M j u_{i t}=\psi_{2}+\psi_{3} \operatorname{Lucbr}_{i t}+\psi_{4} \operatorname{Resluc}_{i t}+\varepsilon_{i t} \\
& V M j u_{i t}=\psi_{7}+\psi_{8} \text { PLubr }_{i t}+\psi_{9} \operatorname{Respl}_{i t}+\varepsilon_{i t}
\end{aligned}
$$

$$
\begin{aligned}
& \text { em que: } \\
& \mathbf{V M j u}_{\mathbf{i t}}=\text { valor de mercado da empresa i } 6 \text { meses após } \\
& \text { o exercício social t; } \\
& \text { Lucbr }_{\text {it }}=\text { lucro líquido da empresa i calculado segundo } \\
& \text { os PCGAs brasileiros para o exercício social } t \text {; } \\
& \text { Resluc }_{\text {it }}=\text { resíduo da equação (13) da empresa i após o } \\
& \text { exercício social } t \text {, equivalente ao lucro líquido } \\
& \text { em US-GAAP não explicado pelo lucro líqui- } \\
& \text { do segundo os PCGAs brasileiros; } \\
& \mathbf{P L b r}_{\text {it }}=\text { patrimônio líquido da empresa i calculado se- } \\
& \text { gundo os PCGAs brasileiros para o exercício } \\
& \text { social } \mathrm{t} \text {; } \\
& \varepsilon_{\mathrm{it}} \quad=\text { termo de erro da regressão. }
\end{aligned}
$$

Ressalte-se que todos os dados a serem utilizados nas regressões (3) a (14) serão tratados em painel (pooled data) não balanceados.

Conforme Greene (1997), três modelos podem ser utilizados para analisar dados em painel (pooled data): utilização de um coeficiente constante, coeficientes randômicos ou coeficientes fixos.

Utilizando um coeficiente comum, seria considerado que todos os dados pertenceriam a uma regressão crosssection, ignorando o fato de que há variáveis de empresas relacionadas com elas em períodos anteriores. Além disso, caso seja utilizado um dos modelos que assumem variações de coeficientes (efeitos fixos ou randômicos), o método mais adequado deverá ser utilizado.

Diante disso, o multiplicador de lagrange proposto por Breusch e Pagan (1980) será utilizado para definição do modelo a ser empregado (efeitos fixos versus randômicos).

\section{RESULTADOS DOS TESTES EMPÍRICOS}

\subsection{Coleta de Dados}

O procedimento utilizado para coleta iniciou-se com a obtenção do banco de dados das empresas listadas na Bolsa de Nova lorque, disponível em seu website ${ }^{5}$. Assim, foi possível a observação de informações acerca de empresas brasileiras com American Depositary Receipts (ADRs) negociados na Bolsa norte-americana desde 1992 até dezembro de 2004. No total, trinta e cinco companhias brasileiras foram identificadas, as quais serviram de ponto inicial para a reunião das variáveis em estudo.
O passo seguinte consistiu na obtenção das variáveis lucro líquido e patrimônio líquido das empresas elaboradas segundo os PCGAs brasileiros. Tais informações foram coletadas por meio da utilização do software Economatica ${ }^{\circledR}$.

Em relação aos lucros líquidos e patrimônios líquidos ajustados aos US-GAAP, esses foram extraídos diretamente dos Relatórios 20-F depositados na SEC por cada uma das empresas. Para tanto, utilizou-se o sistema ED$\mathrm{GAR}^{\mathrm{TM}}$ (Eletronic Data Gathering, Analysis, and Retrieval System), disponível no website da instituição ${ }^{6}$. Para infor-

\footnotetext{
5 http://www.nyse.com. Acessado em 10.02.2005

6 http://www.sec.gov. Acessado em 10.02.2005
} 
mações contábeis divulgadas em dólar, houve a tradução para o real pela taxa de câmbio da data da demonstração contábil'.

Após a análise preliminar dos dados obtidos, considerou-se adequado reduzir o período de estudo para os anos de 1999 a 2004, em função do reduzido número de observações disponíveis entre 1992 e 1998.

Para a coleta das cotações de preços das ações também foi utilizado o software Economatica ${ }^{\circledR}$. As observações foram subdivididas entre ações ordinárias e preferenciais, adotando-se a sugestão de Sarlo Neto (2004), devido aos seus comportamentos similares, o que resultaria em alta correlação das variáveis.

As cotações foram ajustadas aos dividendos distribuídos, utilizando-se um período de tolerância de até 7 dias anteriores. Assim, informações incompletas (ausência de cotação no período) foram descartadas da amostra.

Após todos os procedimentos de agrupamento dos dados, a amostra final utilizada contém 126 observações para ações ordinárias e 137 para ações preferenciais. Após a realização do teste de Breusch e Pagan (1980) para todas as regressões, o modelo de efeitos fixos foi escolhido ${ }^{8}$.

As principais estatísticas descritivas da amostra (média, mediana e desvio-padrão) são apresentadas na Tabela 10

\subsection{Relevância da Informação Contábil segundo os PCGAs brasileiros}

A seguir, a Tabela $2^{9} \mathbf{O}$ apresenta os resultados das equações (5) e (6) para a relevância do lucro líquido e

Tabela 1 Estatísticas descritivas dos dados utilizados na pesquisa

\begin{tabular}{c|c|c|c|c|c|c} 
Variáveis & \multicolumn{2}{|c|}{ Média } & \multicolumn{2}{c}{ Mediana } & \multicolumn{2}{c}{ Desvio-Padrão } \\
\hline & ordinárias & preferenciais & ordinárias & preferenciais & ordinárias & preferenciais \\
VMabit & 0,9160 & 0,9458 & 1,0000 & 0,9596 & 0,4192 & 0,3920 \\
\hline VMjuit & 0,9400 & 0,9542 & 0,9823 & 0,9631 & 0,4526 & 0,3423 \\
\hline Lucbrit & 0,0707 & 0,0751 & 0,0661 & 0,0659 & 0,2194 & 0,2280 \\
\hline Lucusit & 0,0634 & 0,0579 & 0,0487 & 0,0530 & 0,3824 & 0,2811 \\
\hline PLbrit & 1,0239 & 0,9636 & 0,6782 & 0,6836 & 1,6167 & 1,2546 \\
\hline PLusit & 1,0599 & 1,0031 & 0,6410 & 0,6396 & 2,1455 & 1,5483 \\
\hline$\Delta$ Lucit & 0,0073 & 0,0172 & 0,0047 & 0,0068 & 0,2449 & 0,1782 \\
\hline$\Delta$ PLit & $(0,0360)$ & $(0,0395)$ & $(0,0013)$ & $(0,0033)$ & 0,7323 & 0,5271
\end{tabular}

Tabela 2 || Relevância do lucro líquido e do patrimônio líquido elaborado segundo os PCGAs brasileiros

\begin{tabular}{|c|c|c|c|c|c|c|c|}
\hline \multicolumn{4}{|c|}{ Painel A: LUCRO LÍQUIDO } & \multicolumn{4}{|c|}{ Painel B: PATRIMÔNIO LÍQUIDO } \\
\hline \multicolumn{4}{|c|}{ Ações Ordinárias } & \multicolumn{4}{|c|}{ Ações Ordinárias } \\
\hline \multicolumn{3}{|c|}{$\mathrm{VMab}_{\mathrm{it}}=\alpha_{\mathrm{o}}+\alpha_{1} \mathrm{Lucbr}_{\mathrm{it}}+\varepsilon_{\mathrm{it}}$} & (5) & \multicolumn{4}{|c|}{$\mathrm{VMab}_{i \mathrm{t}}=\alpha_{2}+\alpha_{3} \mathrm{PLbr}_{i \mathrm{t}}+\varepsilon_{\mathrm{it}}$} \\
\hline Variáveis & $\alpha_{\circ}$ & $\alpha_{1}$ & $\mathrm{R}^{2}$ & Variáveis & $\alpha_{2}$ & $\alpha_{3}$ & $\mathrm{R}^{2}$ \\
\hline $\mathrm{VMab}_{\text {it }}$ & & 2.059 & 0.07 & $\mathrm{VMab}_{\text {it }}$ & & 0.363 & 0.11 \\
\hline Coeficiente $t$ & & 4.354 & & Coeficiente $t$ & & 4.885 & \\
\hline p-value & & 0.000 & & $p$-value & & 0.000 & \\
\hline \multicolumn{4}{|c|}{ Ações Preferenciais } & \multicolumn{4}{|c|}{ Ações Preferenciais } \\
\hline \multicolumn{3}{|c|}{$\mathrm{VMab}_{\mathrm{it}}=\alpha_{\mathrm{o}}+\alpha_{1} \mathrm{Lucbr}_{\mathrm{it}}+\varepsilon_{\mathrm{it}}$} & (5) & \multicolumn{3}{|c|}{$\mathrm{VMab}_{\mathrm{it}}=\alpha_{2}+\alpha_{3} \mathrm{PLbr}_{\mathrm{it}}+\varepsilon_{\mathrm{it}}$} & \\
\hline Variáveis & $\alpha_{0}$ & $\alpha_{1}$ & $\mathrm{R}^{2}$ & Variáveis & $\alpha_{2}$ & $\alpha_{3}$ & $\mathrm{R}^{2}$ \\
\hline $\mathrm{VMab}_{\text {it }}$ & & 1.113 & 0.01 & $\mathrm{VMab}_{\text {it }}$ & & 0.410 & 0.13 \\
\hline Coeficiente $\mathrm{t}$ & & 3.088 & & Coeficiente $t$ & & 5.015 & \\
\hline$p$-value & & 0.003 & & p-value & & 0.000 & \\
\hline
\end{tabular}

7 A utilização da taxa de câmbio da data da demonstração contábil, e não da ocorrência da transação pode causar distorção, limitação esta que deverá ser levada em conta na análise e generalização dos resultados da pesquisa.

8 Como os coeficientes fixos para cada empresa (interceptos) não constituem o foco das análises realizadas na pesquisa, estes não são apresentados nas tabelas, visando simplificar seu layout.

9 As tabelas foram organizadas em dois painéis: o Painel A refere-se ao lucro líquido e o Painel B ao patrimônio líquido. Os resultados das regressões são apresentados para ações ordinárias e preferenciais, separadamente. As variáveis de cada regressão estão descritas na seção de Metodologia de Pesquisa. 
do patrimônio líquido elaborados segundo os PCGAs brasileiros.

A análise da Tabela 2 indica que tanto o lucro líquido quanto o patrimônio líquido elaborados segundo os PCGAs brasileiros apresentam relativa relevância para o mercado, com coeficientes estatisticamente significantes a 99\%.

Os achados são similares aos de Lopes (2001) e pode-se inferir que as informações contábeis elaboradas segundo os PCGAs brasileiros são relevantes para o mercado, mesmo que essas divulguem informações baseadas em US-GAAP.

Um fator que pode justificar sua relevância seria o da oportunidade da informação, dado que as demonstrações contábeis são divulgadas até 30 de abril no Brasil, prazo inferior ao exigido pela SEC para o depósito das informações segundo os US-GAAP, que é de 30 de junho.

A maior relevância do patrimônio líquido em relação ao lucro líquido pode ser justificada pelo enquadramento do Brasil como um país code-law, cujas características são de possuir um modelo orientado principalmente para bancos e para tributação, e não para o mercado de capitais.

Além disso, o controle acionário é altamente concentrado (SILVA e LEAL, 2004) e como conseqüência, os lucros líquidos contábeis perdem relevância para os investidores ao contrário do que ocorre com os patrimônios líquidos, dado que esses consistiriam em uma proxy para o valor de liquidação das empresas.

\subsection{Relevância das Informações em US-GAAP divulgadas em junho e sua Comparação com as Informações segundo os PCGAs brasileiros na mesma Data}

A Tabela 3 apresentada os resultados das equações (7) e (8), acerca da relevância do lucro líquido e do patrimônio líquido elaborados conforme os US-GAAP.
Mesmo com coeficientes estatisticamente significativos a $99 \%$ de confiança, percebe-se que os resultados da equação (7) referentes à relevância dos lucros líquidos elaborados em conformidade com os US-GAAP apresentam poderes explicativos $\left(\mathrm{R}^{2}\right)$ nulos, tanto para ações ordinárias quanto para preferenciais.

Em parte, uma das razões pode estar ligada às evidências apresentadas por Tukamoto (2004), de que o nível de gerenciamento de resultados (earnings management) das empresas brasileiras com ADRs negociados na Bolsa de Nova lorque, mesmo em US-GAAP, são similares aos de empresas que não possuem tais títulos.

Outro fator seria o de que as empresas utilizadas na amostra ainda possuem controle acionário concentrado (SILVA e LEAL, 2004), o que implica a redução da capacidade de redução de assimetria informacional do lucro líquido ajustado aos US-GAAP.

Em relação ao patrimônio líquido elaborado segundo os US-GAAP, os resultados referentes à equação (8) apontam para coeficientes estatisticamente significantes a $99 \%$, permitindo a observação de que há indicativos para uma maior relevância do patrimônio líquido conforme os USGAAP para acionistas preferenciais, se comparados aos ordinários.

Entretanto, conforme discutido na revisão da literatura, espera-se que a informação contábil segundo os PCGAs brasileiros seja superior à informação elaborada conforme os US-GAAP, similarmente aos resultados apresentados por Chan e Seow (1996). Tal fato seria justificado pela divulgação das informações segundo o conjunto local (no caso, o brasileiro) ser feita dois meses antes do que o conjunto norte-americano.

Dessa forma, as variáveis em US-GAAP já seriam, ao menos em parte, antecipadas em abril pela divulgação daquelas segundo os PCGA brasileiros.

Tabela 3 | Relevância do lucro líquido e do patrimônio líquido elaborados segundo os US-GAAP

\begin{tabular}{|c|c|c|c|c|c|c|c|}
\hline \multicolumn{4}{|c|}{ Painel A: LUCRO LÍQUIDO } & \multicolumn{4}{|c|}{ Painel B: PATRIMÔNIO LÍQUIDO } \\
\hline \multicolumn{4}{|c|}{ Ações Ordinárias } & \multicolumn{4}{|c|}{ Ações Ordinárias } \\
\hline \multicolumn{3}{|c|}{$\mathrm{VMju}_{\mathrm{it}}=\lambda_{\mathrm{o}}+\lambda_{1}$ Lucus $_{\mathrm{it}}+\varepsilon_{\mathrm{it}}$} & (7) & \multicolumn{3}{|c|}{$\mathrm{VMju}_{\mathrm{it}}=\lambda_{2}+\lambda_{3} \mathrm{PLus}_{\mathrm{it}}+\varepsilon_{\mathrm{it}}$} & (8) \\
\hline Variáveis & $\lambda_{\mathrm{o}}$ & $\lambda_{1}$ & $\mathrm{R}^{2}$ & Variáveis & $\lambda_{2}$ & $\lambda_{3}$ & $\mathrm{R}^{2}$ \\
\hline $\mathrm{VMju}_{\mathrm{it}}$ & & 1.090 & 0.00 & $\mathrm{VMju}_{\mathrm{it}}$ & & 0.245 & 0.02 \\
\hline Coeficiente $\mathrm{t}$ & & 2.898 & & Coeficiente $\mathrm{t}$ & & 3.511 & \\
\hline$p$-value & & 0.005 & & $\mathrm{p}$-value & & 0.001 & \\
\hline \multicolumn{4}{|c|}{ Ações Preferenciais } & \multicolumn{4}{|c|}{ Ações Preferenciais } \\
\hline \multicolumn{3}{|c|}{$\mathrm{VMju}_{\mathrm{it}}=\lambda_{\mathrm{o}}+\lambda_{1}$ Lucus $_{\mathrm{it}}+\varepsilon_{\mathrm{it}}$} & (7) & \multicolumn{3}{|c|}{$\mathrm{VMju}_{\mathrm{it}}=\lambda_{2}+\lambda_{3} \mathrm{PLus}_{\mathrm{it}}+\varepsilon_{\mathrm{it}}$} & \\
\hline Variáveis & $\lambda_{\mathrm{o}}$ & $\lambda_{1}$ & $\mathrm{R}^{2}$ & Variáveis & $\lambda_{2}$ & $\lambda_{3}$ & $R^{2}$ \\
\hline $\mathrm{VMju}_{\mathrm{it}}$ & & 1.168 & 0.00 & $\mathrm{VMju}_{\mathrm{it}}$ & & 0.285 & 0.05 \\
\hline Coeficiente $\mathrm{t}$ & & 2.984 & & Coeficiente $\mathrm{t}$ & & 3.779 & \\
\hline$p$-value & & 0.004 & & $p$-value & & 0.000 & \\
\hline
\end{tabular}


Assim, é necessária a comparação entre os resultados da Tabela 3 e os apresentados na Tabela $4 \mathbf{0}$, que levam em conta as variáveis contábeis elaboradas segundo o modelo contábil brasileiro.

Na Tabela 4 são apresentados os resultados das equações (9) e (10) referentes à associação entre o lucro líquido e o patrimônio líquido, respectivamente, ao valor de mercado da empresa em junho.

Os dados da Tabela 4 indicam que o lucro líquido apresenta relevância para acionistas ordinários, o mesmo não acontecendo para os preferenciais. Dessa forma, corroborase o fato de que o resultado segundo o conjunto de princípios contábeis brasileiros teria igual ou maior relevância do que o elaborado em conformidade com o norte-americano.

Já em relação ao patrimônio líquido, percebe-se que as regressões são estatisticamente significativas a $98 \%$ e que seus poderes explicativos indicam para uma maior relevância das informações elaboradas segundo os PCGAs brasileiros em comparação com os norte-americanos. Os patrimônios líquidos divulgados em junho (US-GAAP) teriam sua relevância menor porque parte de seu conteúdo informacional já estaria presente nas demonstrações contábeis publicadas em abril.

Os resultados, portanto, são coerentes com os achados de Chan e Seow (1996). Porém, conforme discutido no capítulo três, os autores não contemplaram a possibilidade de que as demonstrações elaboradas segundo os USGAAP apresentarem um mix entre conteúdo informacional antigo (disponível em abril) e novo, o qual não poderia ser antecipado pelo mercado.

\subsection{Relevância dos Ajustes aos US-GAAP}

Os resultados apresentados nas seções anteriores (4.2 e 4.3) permitiram avaliar que as informações segundo os
PCGAs brasileiros são relevantes para o mercado e possuem igual ou maior poder explicativo do que aquelas divulgadas em junho elaboradas segundo os US-GAAP.

Nessa seção, são apresentados os resultados dos ajustes aos US-GAAP, os quais foram definidos no presente trabalho como sendo as proxies para o cálculo das informações que seriam consideradas como novas ao mercado ${ }^{10}$.

A seguir, apresentam-se os resultados das regressões para testar a relevância dos ajustes do lucro líquido e do patrimônio líquido aos US-GAAP. A Tabela 50 consiste no cálculo da parcela (resíduo da regressão) do lucro líquido e do patrimônio líquido elaborados segundo o modelo norte-americano não explicada pelo brasileiro.

Na Tabela $6 \bigcirc$ são apresentados os resultados da regressão para avaliar a relevância do ajuste do lucro líquido e ao patrimônio líquido para os US-GAAP.

$A$ análise da Tabela 6 indica que o ajuste aos US-GAAP efetuado no lucro líquido não confere informação relevante ao mercado. Os coeficientes não são estatisticamente significantes a $99 \%$ e nem a $95 \%$. Além disso, o poder explicativo $\left(R^{2}\right)$ é nulo.

Conforme discutido anteriormente, a alta concentração acionária (SILVA e LEAL: 2004) e o nível de gerenciamento de resultados (TUKAMOTO: 2004) podem ser razões citadas para explicar a irrelevância encontrada para os ajustes aos US-GAAP. Portanto, as informações disponibilizadas em abril conforme o modelo brasileiro seriam fonte suficiente de informação para o mercado.

Em relação ao patrimônio líquido, a análise da Tabela 6 indica que os ajustes aos US-GAAP efetuados são relevantes para o mercado, interpretando-se que estes refletem informações significativas e que não seriam antecipadas em abril.

Tabela 4 || Relevância do lucro líquido e do patrimônio líquido elaborados segundo os PCGAs brasileiros em 30 de junho

\begin{tabular}{|c|c|c|c|c|c|c|c|}
\hline \multicolumn{4}{|c|}{ Painel A: LUCRO LíQUIDO } & \multicolumn{4}{|c|}{ Painel B: PATRIMÔNIO LÍQUIDO } \\
\hline \multicolumn{4}{|c|}{ Ações Ordinárias } & \multicolumn{4}{|c|}{ Ações Ordinárias } \\
\hline \multicolumn{3}{|c|}{$\mathrm{VMju}_{\mathrm{it}}=\varphi_{\mathrm{o}}+\varphi_{1} \mathrm{Lucbr}_{\mathrm{it}}+\varepsilon_{\mathrm{it}}$} & (9) & \multicolumn{3}{|c|}{$\mathrm{VMju}_{\mathrm{it}}=\varphi_{2}+\varphi_{3} \mathrm{PLbr}_{\mathrm{it}}+\varepsilon_{\mathrm{it}}$} & $(10)$ \\
\hline Variáveis & $\phi_{\circ}$ & $\phi_{1}$ & $\mathrm{R}^{2}$ & Variáveis & $\phi_{2}$ & $\phi_{3}$ & $\mathrm{R}^{2}$ \\
\hline $\mathrm{VMju}_{\mathrm{it}}$ & & 2.217 & 0.04 & $\mathrm{VMju}_{\mathrm{it}}$ & & 0.440 & 0.11 \\
\hline Coeficiente t & & 3.785 & & Coeficiente $t$ & & 4.874 & \\
\hline$p$-value & & 0.000 & & p-value & & 0.000 & \\
\hline \multicolumn{4}{|c|}{ Ações Preferenciais } & \multicolumn{4}{|c|}{ Ações Preferenciais } \\
\hline \multicolumn{3}{|c|}{$\mathrm{VMju}_{\mathrm{it}}=\varphi_{\mathrm{o}}+\varphi_{1} \mathrm{Lucbr}_{\mathrm{it}}+\varepsilon_{\mathrm{it}}$} & 9) & \multicolumn{3}{|c|}{$\mathrm{VMju}_{\mathrm{it}}=\varphi_{2}+\varphi_{3} \mathrm{PLbr}_{\mathrm{it}}+\varepsilon_{\mathrm{it}}$} & \\
\hline Variáveis & $\phi_{\circ}$ & $\phi_{1}$ & $\mathrm{R}^{2}$ & Variáveis & $\phi_{2}$ & $\phi_{3}$ & $R^{2}$ \\
\hline $\mathrm{VMju}_{\mathrm{it}}$ & & 1.015 & 0.00 & $\mathrm{VMju}_{\mathrm{it}}$ & & 0.452 & 0.12 \\
\hline Coeficiente t & & 2.448 & & Coeficiente $\mathrm{t}$ & & 4.854 & \\
\hline$p$-value & & 0.016 & & $p$-value & & 0.000 & \\
\hline
\end{tabular}


Tabela 5 Cálculo da informação em US-GAAP referente ao lucro líquido e ao patrimônio líquido não contido nas informações divulgadas em abril (PCGAs brasileiros)

\begin{tabular}{|c|c|c|c|c|c|c|c|}
\hline \multicolumn{4}{|c|}{ Painel A: LUCRO LíQUIDO } & \multicolumn{4}{|c|}{ Painel B: PATRIMÔNIO LÍQUIDO } \\
\hline \multicolumn{4}{|c|}{ Ações Ordinárias } & \multicolumn{4}{|c|}{ Ações Ordinárias } \\
\hline \multicolumn{3}{|c|}{ Lucus $_{\text {it }}=\psi_{0}+\psi_{1}$ Lucbr $_{i t}+\varepsilon_{i t}$} & $(11)$ & \multicolumn{4}{|c|}{ PLus $_{\text {it }}=\psi_{5}+\psi_{6} \mathrm{PLbr}_{\mathrm{it}}+\varepsilon_{\mathrm{it}}$} \\
\hline Variáveis & $\psi_{\circ}$ & $\psi_{1}$ & $R^{2}$ & Variáveis & $\psi_{5}$ & $\psi_{6}$ & $R^{2}$ \\
\hline Lucbr $_{\text {it }}$ & & 1.239 & 0.64 & $\mathrm{PLbr}_{\text {it }}$ & & 1.308 & 0.95 \\
\hline Coeficiente $t$ & & 11.899 & & Coeficiente $\mathrm{t}$ & & 34.184 & \\
\hline p-value & & 0.000 & & $p$-value & & 0.000 & \\
\hline \multicolumn{4}{|c|}{ Ações Preferenciais } & \multicolumn{4}{|c|}{ Ações Preferenciais } \\
\hline \multicolumn{3}{|c|}{ Lucus $_{\text {it }}=\psi_{0}+\psi_{1}$ Lucbr $_{i t}+\varepsilon_{i t}$} & & \multicolumn{4}{|c|}{ PLus $_{\text {it }}=\psi_{5}+\psi_{6} \mathrm{PLbr}_{\mathrm{it}}+\varepsilon_{\mathrm{it}}$} \\
\hline Variáveis & $\psi_{\circ}$ & $\psi_{1}$ & $\mathrm{R}^{2}$ & Variáveis & $\psi_{5}$ & $\psi_{6}$ & $\mathrm{R}^{2}$ \\
\hline Lucbr $_{\text {it }}$ & & 0.652 & 0.51 & $\mathrm{PLbr}_{\text {it }}$ & & 1.224 & 0.95 \\
\hline Coeficiente $\mathrm{t}$ & & 8.179 & & Coeficiente $\mathrm{t}$ & & 32.514 & \\
\hline p-value & & 0.000 & & $p$-value & & 0.000 & \\
\hline
\end{tabular}

Tabela 6 Relevância dos ajustes aos US-GAAP efetuados no lucro líquido

\begin{tabular}{|c|c|c|c|c|c|c|c|c|c|}
\hline \multicolumn{5}{|c|}{ Painel A: LUCRO LÍQUIDO } & \multicolumn{5}{|c|}{ Painel B: PATRIMÔNIO LÍQUIDO } \\
\hline \multicolumn{5}{|c|}{ Ações Ordinárias } & \multicolumn{5}{|c|}{ Ações Ordinárias } \\
\hline \multicolumn{4}{|c|}{$\mathrm{VMju}_{\mathrm{it}}=\psi_{2}+\psi_{3} \mathrm{Lucbr}_{\mathrm{it}}+\psi_{4}$ Resluc $_{\mathrm{it}}+\varepsilon_{\mathrm{it}}$} & $(13)$ & \multicolumn{5}{|c|}{$\mathrm{VMju}_{\mathrm{it}}=\psi_{7}+\psi_{8} \mathrm{PLbr}_{\mathrm{it}}+\psi_{9} \operatorname{Respl}_{\mathrm{it}}+\varepsilon_{\mathrm{it}}$} \\
\hline Variáveis & $\psi_{2}$ & $\psi_{3}$ & $\psi_{4}$ & $\mathrm{R}^{2}$ & Variáveis & $\psi_{7}$ & $\psi_{8}$ & $\psi_{9}$ & $\mathrm{R}^{2}$ \\
\hline Lucbr $_{\text {it }}$ & & 2.217 & 0.047 & 0.03 & $\mathrm{PLbr}_{\mathrm{it}}$ & & 0.440 & -0.877 & 0.23 \\
\hline Coeficiente $\mathrm{t}$ & & 3.765 & 0.082 & & Coeficiente $\mathrm{t}$ & & 5.222 & -3.833 & \\
\hline$p$-value & & 0.000 & 0.935 & & p-value & & 0.000 & 0.000 & \\
\hline \multicolumn{5}{|c|}{ Ações Preferenciais } & \multicolumn{5}{|c|}{ Ações Preferenciais } \\
\hline \multicolumn{4}{|c|}{$\mathrm{VMju}_{\mathrm{it}}=\psi_{2}+\psi_{3}$ Lucbr $_{\mathrm{it}}+\psi_{4}$ Resluc $_{\mathrm{it}}+\varepsilon_{\mathrm{it}}$} & & \multicolumn{5}{|c|}{$\mathrm{VMju}_{\mathrm{it}}=\psi_{7}+\psi_{8} \mathrm{PLbr}_{\mathrm{it}}+\psi_{9} \operatorname{Respl}_{\mathrm{it}}+\varepsilon_{\mathrm{it}}$} \\
\hline Variáveis & $\psi_{2}$ & $\psi_{3}$ & $\psi_{4}$ & $\mathrm{R}^{2}$ & Variáveis & $\psi_{7}$ & $\psi_{8}$ & $\psi_{9}$ & $\mathrm{R}^{2}$ \\
\hline Lucbr $_{\text {it }}$ & & 1.015 & 0.921 & 0.00 & $\mathrm{PLbr}_{\text {it }}$ & & 0.452 & -0.568 & 0.16 \\
\hline Coeficiente $\mathrm{t}$ & & 2.448 & 1.835 & & Coeficiente $\mathrm{t}$ & & 4.963 & -2.403 & \\
\hline p-value & & 0.016 & 0.069 & & $p$-value & & 0.000 & 0.018 & \\
\hline
\end{tabular}

Todos os coeficientes são estatisticamente significantes a $98 \%$ e $95 \%$. Há indícios também de que os poderes explicativos das regressões apresentadas na Tabela 6 sejam maiores do que aqueles se levando em consideração apenas as informações segundo os PCGAs brasileiros em 30 de junho.

Em relação às subamostras, os dados apontam para uma maior relevância das informações acerca do patrimônio líquido para acionistas ordinários (23\%) em relação aos preferenciais (16\%).

A característica do mercado brasileiro, cuja principal fonte de financiamento é via Bancos, poderia explicar tais resultados. A Tabela 1 evidencia que o patrimônio líquido é menor, em média, quando elaborado segundo US-GAAP, indicando que o modelo norte-americano seria mais conservador, se comparado ao brasileiro.

Sendo assim, sua relevância poderia estar atrelada à característica de ser uma proxy mais conservadora do valor de liquidação da empresa e também para acompanhamento de covenants (índice dívida/patrimônio líquido, por exemplo) para os credores. 


\section{CONSIDERAC̣ÕES FINAIS}

O presente estudo foi realizado para avaliar a relevância das informações contábeis ajustadas aos US-GAAP realizados por empresas brasileiras que possuem ADRs negociados na Bolsa de Nova lorque.

O trabalho procura contribuir para o atual debate em relação aos benefícios ou não do movimento em prol da convergência, conforme apresentado por Barth et al. (1999) em seu modelo analítico. Além disso, amplia a literatura existente ao apresentar resultados sobre empresas brasileiras, as quais ocupam a terceira posição na Bolsa de Nova lorque em termos de quantidade de empresas com ADRs negociados por país.

Os resultados obtidos na seção 4.2 indicam que a informação contábil elaborada com base no conjunto de princípios brasileiros corresponde à informação útil e relevante para o mercado, expandindo as conclusões da pesquisa realizada por Lopes (2001), já que o patrimônio líquido possui maior relevância que o lucro líquido, mesmo para empresas com títulos negociados em mercados estrangeiros.

Na seção 4.3, as regressões apontaram para maior relevância das informações segundo o conjunto de princípios contábeis locais das empresas em comparação ao norteamericano. Apesar de estatisticamente significativas, as informações em US-GAAP seriam menos relevantes, pois parte significativa estaria contida ou seria antecipada nas informações divulgadas em abril. O resultado é similar ao encontrado por Chan e Seow (1996).

Diante dos dados obtidos nas seções 4.2 e 4.3 , foi possível avaliar se os ajustes US-GAAP realizados no lucro líquido e no patrimônio líquido consistiriam em informações novas e relevantes para o mercado de capitais. Os dados apresentados na seção 4.4 evidenciam que o ajuste efetuado no patrimônio líquido é relevante para o mercado, diferentemente do que ocorre com o lucro líquido.

Em suma, a análise agregada dos resultados indica que: (a) a informação contábil segundo os princípios brasileiros é relevante para o mercado; (b) as informações em USGAAP possuem conteúdo informacional já existente e/ou antecipado em abril; (c) os ajustes aos US-GAAP são relevantes para o mercado, em relação ao patrimônio líquido, mas não no tocante ao lucro líquido.
Espera-se que os resultados obtidos contribuam para auxiliar os organismos reguladores em sua avaliação sobre uma possível inserção do Brasil no movimento atual de convergência de normas contábeis.

As limitações do trabalho, discutidas a seguir, permitem insights para o desenvolvimento de novas pesquisas. A resposta afirmativa em relação à relevância dos ajustes aos USGAAP efetuados no patrimônio líquido e da irrelevância em relação ao lucro, já enseja uma série de questionamentos.

A relação entre a estrutura de governança das empresas e a Contabilidade poderia ser um ponto de partida. Além disso, outras características, como setor e complexidade das operações desenvolvidas pela companhia, por exemplo, poderiam ser estudadas. Dessa forma, seriam contempladas variáveis cross-country e within-country, ampliando a robustez dos resultados empíricos.

Em relação aos dados utilizados, a amostra não considerou a existência de diferenças entre práticas e normas contábeis em cada setor. Há setores altamente regulamentados, como o de telecomunicações e de serviços financeiros (Bancos). Além disso, há empresas públicas ou de economia mista tratadas indistintamente como empresas privadas. Nesse sentido, novas pesquisas poderiam ser desenvolvidas considerando o controle acionário e também a estrutura de governança de cada empresa.

As regressões utilizadas, também, podem incluir variáveis de controle, tais como ciclos econômicos, tamanho da empresa, número de anos em que a empresa está listada em duas Bolsas e instrumentos de governança corporativa utilizados. O trabalho também pode ser desenvolvido para países de blocos econômicos, tais como o Mercosul, ou ainda para empresas de países emergentes relevantes, como Rússia ou Índia.

Por fim, vale ressaltar que os achados desta pesquisa são concernentes à metodologia e à base de dados utilizada e inferências sobre impactos futuros deverão ser feitas de maneira cautelosa e com fundamentação em outras pesquisas. Espera-se que este trabalho seja considerado relevante não só pelos seus achados, mas pela abertura de novos caminhos para a pesquisa e para o desenvolvimento da Contabilidade e seu papel como informação útil para a tomada de decisão.

\section{Referências Bibliográficas}

ALFORD, Andrew; JONES, Jennifer; LEFTWICH, Richard; ZMIJEWSKI, Mark. The Relative Informativeness of Accounting Disclosures in Different Countries. Journal of Accounting Research, vol. 31, Supplement, pp. 183-223, 1993.

AMIR, Eli; HARRIS, Trevor S.; VENUTI, Elizabeth K. A Comparison of the Value-Relevance of U.S. versus Non-U.S. GAAP Accounting Measures Using Form 20-F Reconciliations. Journal of Accounting Research, vol. 31 Supplement, pp. 230-264, 1993.

BALL, R.; KOTHARI, S. P.; ROBIN, A. The Effect of International Institutional Factors on Properties of Accounting Earnings. Journal of Accounting and Economics, vol. 29, pp. 1-51, 2000.

BANDYOPADHYAY, Sati P.; HANNA, Douglas; RICHARDSON, Gordon. Capital Market Effects of U.S.-Canada GAAP Differences. Journal of Accounting Research, vol. 32, n. 2, pp. 262-277, Autumn 1994. 
BARTH, Mary E.; BEAVER, William H.; LANDSMAN, Wayne R. The Relevance of the Value Relevance Literature for Financial Accounting Standard Setting: Another View. Journal of Accounting \& Economics, vol. 31, n. 1-3, pp. 77-118, September 2001.

; CLINCH, Greg. International Accounting Differences and Their Relation to Share Prices: Evidence from U.K., Australian, and Canadian Firms. Contemporary Accounting Research, vol. 13, n. 1, pp. 135-170, Spring 1996.

; SHIBANO, Toshi. International Accounting Harmonization and Global Equity Markets. Journal of Accounting and Economics, n. 26, pp. 201-235, 1999.

BHUSHAN, Ravi; LESSARD, Donald R. Coping With International Accounting Diversity: Fund Managers' Views on Disclosure, Reconciliation, and Harmonization. Journal of International Financial Management \& Accounting, vol. 4, n. 2, pp. 149-164, Summer 1992.

BREUSCH, T. S.; PAGAN, A. R. The Lagrange Multiplier Test and Its Application to Model Specification in Econometrics. Review of Economic Studies, vol. 47, pp. 239-254, 1980.

BROWN, S.; LO, K.; LYS, T. Use of $\mathrm{R}^{2}$ in accounting research: measuring changes in value relevance over the last four decades. Journal of Accounting and Economics, vol. 28, pp. 83-115, 1999.

CHAN, Kam C.; SEOW, Gim S. The Association Between Stock Returns and Foreign GAAP Earnings Versus Earnings Adjusted to U.S. GAAP. Journal of Accounting and Economics, vol. 21, pp. 139-158, 1996.

CHOI, Frederick D.S.; LEVICH, Richard M. Behavioral Effects of International Accounting Diversity. Accounting Horizons, vol. 5, n. 1, pp. 1-13, Jun 1991.

COLLINS, D. W.; MAYDEW, E. L.; WEISS, I. S. Changes in Value-Relevance of Earnings and Book Values over the Past Forty Years. Journal of Accounting and Economics, 24, pp. 39-67, 1997.

DAVIS-FRIDAY, Paquita Y.; RIVERA, Juan M. Inflation Accounting and 20-F Disclosures: Evidence from Mexico. Accounting Horizons, vol. 14, n. 2, pp. 113-135, June 2000.

FELTHAM, Gerald A.; OHLSON, James A. Valuation and Clean Surplus Accounting for Operating and Financial Activities. Contemporary Accounting Research, vol. 11, n. 2, pp. 689-732, Spring 1995.

pp. 209-234, Autumn 1996.

. Uncertainty Resolution and the Theory of Depreciation Measurement. Journal of Accounting Research, vol. 34, n. 2,

FRIEDMAN, Milton. The Methodology of Positive Economics. In: Essays In Positive Economics. Chiagado: University of Chicago Press, 1966. pp. 3-16, 30-43.

GREENE, William H. Econometric Analysis. $3^{\text {rd }}$ ed. Prentice Hall, New Jersey: 1997. 1075 pp.

HARRIS, T. S., LẢNG, M.; MÖLLER, H. P. The Value Relevance of German Accounting Measures: An Empirical Analysis. Journal of Accounting Research, vol. 32, no. 2, Autumn 1994.

HAVERTY, John L. Are IFRS and U.S. GAAP Converging? Some Evidence from People's Republic of China Companies Listed on New York Stock Exchange. In: American Accounting Association Mid-Atlantic Region Meeting. Anais... Arlington, Washington DC, 2004.

LAPORTA, Rafel; LOPES-DE-SILANES, Florêncio; SHLEIFER, Andrei \& VISHNY, Robert. Legal Determinants of External Finance. The Journal of Finance, vol. 52, n. 3, pp. 1131-1150, 1997.

Law \& Finance. The Journal of Political Economy, vol. 106, n. 6, pp. 1113-1155, 1998

Investor Protection and Corporate Governance. Journal of Financial Economics, vol. 58, n. 1-2, pp. 3-27, Oct/Nov 2000.

LEFTWICH, R. Market Failure Fallacies and Accounting Information. Journal of Accounting and Economics, n. 2, pp. 193-211, 1980.

LEUZ, Christian. IAS versus U.S. GAAP: Information Assymetry-based Evidence from Germany's New Market. Journal of Accounting Research, vol. 41, n. 3, pp. 445-472, June 2003.

LOPES, Alexsandro Broedel. A Relevância da Informação Contábil para o Mercado de Capitais: O Modelo de Ohlson Aplicado à BOVESPA. Tese 2001 (Doutorado em Controladoria e Contabilidade). Faculdade de Economia, Administração e Contabilidade da Universidade de São Paulo são Paulo.

MEEK, Gary K. U.S.. Securities Market Responses to Alternative Earnings Disclosures of Non-U.S. Multinational Corporations. The Accounting Review, vol. LVIII, n. 2, pp. 394-402, April 1983.

; SAUDAGARAN, Shahrokh M. A Review of Research on the Relationship Between International Capital Markets and Financial Reporting by Multinational Firms. Journal of Accounting Literature, vol. 16, pp. 127-159, 1997.

; THOMAS, Wayne B.. A Review of Markets-Based International Accounting Research. Journal of International Accounting Research, vol. 3, n. 1, pp. 21-41, 2004.

OHLSON, James. A. Earnings, Book Values and Dividends in Equity Valuation. Contemporary Accounting Research, vol. 11, n. 2, pp. 661-687, 1995.

POPE, Peter F.. Discussion of A Comparison of the Value-Relevance of U.S. versus Non-U.S. GAAP Accounting Measures Using Form 20-F Reconciliations. Journal of Accounting Research, vol. 31, Supplement, pp. 265-275, 1993.

POWNALL, Grace. Discussion of The Relative Informativeness of Accounting Disclosures in Different Countries. Journal of Accounting Research, vol. 31, Supplement, pp. 224-229, 1993.

SARLO NETO, Alfredo. A Reação dos Preços das Ações à Divulgação dos Resultados Contábeis: Evidências Empíricas Sobre a Capacidade Informacional da Contabilidade no Mercado Acionário Brasileiro. Dissertação 2004 (Mestrado Profissional em Ciências Contábeis). Fundação Instituto Capixaba de Pesquisas em Contabilidade, Economia e Finanças, Vitória, Espírito Santo. 
SILVA, André Carvalhal da; LEAL, Ricardo P. C.. Corporate Governance, Market Valuation and Dividend Policy in Brazil. Working Paper, Universidade Federal do Rio de Janeiro, 17 pp., 2004.

TUKAMOTO, Yhurika Sandra. Contribuição ao Estudo do "Gerenciamento" de Resultados - Uma Comparação entre as Companhias Abertas Brasileiras Emissoras de ADRs e Não Emissoras de ADRs. Dissertação 2004 (Mestrado em Controladoria e Contabilidade). Faculdade de Economia, Administração e Contabilidade da Universidade de São Paulo, São Paulo.

VUONG, Quang H.. Likelihood Ratio Tests for Model Selection and Non-Nested Hypotheses. Econometrica, vol. 57, n. 2, pp. $307-333$, March 1989. WILBER, Charles K. The Chicago School: Positivism or Ideal Type. Journal of Economic Issues, vol. 9, n. 4, pp. 665-679, December 1975.

ZEFF, Stephen A.. The Rise of "Economic Consequences" in ZEFF, Stephen A.; DHARAN, Bala. Reading and Notes on Financial Accounting: Issues and Controversies. $5^{\text {th }}$ ed. Irwin/McGraw-Hill, $1994.672 \mathrm{pp}$.

\section{NOTA - Endereço dos autores}

FUCAPE

Av. Fernando Ferrari, 1358 - Goiabeiras

Vitória - ES

29075-010
Universidade de São Paulo

Faculdade de Economia, Administração e Contabilidade

Departamento de Contabilidade e Atuária

Av. Prof. Luciano Gualberto, 908 - prédio 3

São Paulo - SP

05508-900 